\title{
Multiple Periodic Solutions of an Impulsive Population System with Delays
}

\author{
Wenbo Zhao, ${ }^{1}$ Caochuan $\mathrm{Ma}^{2}$ and Lijun $\mathrm{Chen}^{3}$ \\ ${ }^{1}$ School of Physics and Information, Tianshui Normal University, Tianshui, Gansu 741000, China \\ ${ }^{2}$ School of Mathematics and Statistics, Tianshui Normal University, Tianshui, Gansu 741000, China \\ ${ }^{3}$ Department of Mathematics, Zhejiang Normal University, Jinhua, Zhejiang 321004, China
}

Correspondence should be addressed to Caochuan Ma; ccmamath@gmail.com

Received 15 March 2014; Accepted 29 March 2014; Published 11 May 2014

Academic Editor: Yonghui Xia

Copyright (C) 2014 Wenbo Zhao et al. This is an open access article distributed under the Creative Commons Attribution License, which permits unrestricted use, distribution, and reproduction in any medium, provided the original work is properly cited.

\begin{abstract}
A nonautonomous plant-hare model with impulse is considered. By using the continuation theorem of coincidence degree theory, we present an easily verifiable sufficient condition on the existence of multiple periodic solutions. Though Gao et al. (2014) considered the periodic solutions of plant-hare model, such model with impulses and delay has not been studied in previous paper.
\end{abstract}

\section{Introduction}

In recent years, applications of theory differential equations in mathematical ecology have developed rapidly. Various mathematical models have been proposed in the study of population dynamics (see [1-13]). Recently, Gao et al. [5] considered a nonautonomous plant-hare dynamical system with a toxin-determined functional response given by

$$
\begin{gathered}
\dot{N}(t)=r(t) N(t)\left[1-\frac{N(t)}{K}\right]-C(N(t)) P(t), \\
\dot{P}(t)=B(t) C(N(t)) P(t)-d(t) P(t), \\
C(N(t))=f(N(t))\left[1-\frac{f(N(t))}{4 G}\right], \\
f(N(t))=\frac{e \delta N(t)}{1+h e \delta N(t)}
\end{gathered}
$$

where $N(t)$ denotes the density of plant at time $t, P(t)$ denotes the herbivore biomass at time $t, r(t)$ is the plant intrinsic growth rate at time $t, d(t)$ is the per capita rate of herbivore death unrelated to plant toxicity at time $t$, and $B(t)$ is the conversion rate at time $t$. $e$ is the encounter rate per unit plant, $\delta$ is the fraction of food items encountered that the herbivore ingests, $K$ is the carrying capacity of plant, $G$ measures the toxicity level, and $h$ is the time for handing one unit of plant. $r(t), d(t)$, and $B(t)$ are continuously positive periodic functions with period $\omega$ and $e, \delta, K, G$, and $h$ are five positive real constants.

However, birth of many species is an annual birth pulse; for having more accurate description to the system, we need to consider incorporating the impulsive effect into the differential equations. For more biological view of impulses, one can refer to [14-17]. To describe to a system more accurately, we should consider the following impulsive system with delays:

$$
\begin{aligned}
\dot{N}(t)= & N(t)\left[r(t)\left(1-\frac{N\left(t-\tau_{1}\right)}{K}\right)\right. \\
& \left.-\frac{4 G e \delta P(t)+(4 G h-1) e^{2} \delta^{2} N(t) P(t)}{4 G(1+h e \delta N(t))^{2}}\right], \\
\dot{P}(t)= & P(t) \quad \times\left[\frac{4 G e \delta B(t) N\left(t-\tau_{2}\right)+(4 G h-1) e^{2} \delta^{2} B(t) N^{2}(t)}{4 G(1+h e \delta N(t))^{2}}\right. \\
& -d(t)], \quad t \neq t_{k} ;
\end{aligned}
$$




$$
\begin{gathered}
\Delta N\left(t_{k}\right)=N\left(t_{k}^{+}\right)-N\left(t_{k}^{-}\right)=c_{1 k} N\left(t_{k}\right), \\
\Delta P\left(t_{k}\right)=P\left(t_{k}^{+}\right)-P\left(t_{k}^{-}\right)=c_{2 k} P\left(t_{k}\right), \quad t=t_{k}, k=1,2, \ldots,
\end{gathered}
$$

where the assumptions on $r, d, B, e, \delta, K, G$, and $h$ are the same as before; $c_{j k} \in(-1, \infty)(j=1,2, k \in \mathbb{N}=1,2, \ldots)$, $\left\{t_{k}\right\} \in \mathbb{N}$ is a strictly increasing sequence with $t_{1}>0$ and $\lim _{k \rightarrow \infty} t_{k}=\infty$. We further assume that there exists a $q \in \mathbb{N}$ such that $c_{j(k+q)}=c_{j k}(j=1,2)$ and $t_{k+q}=t_{k}+\omega$ for $k \in \mathbb{N}$.

Without loss of generality, we will assume $t_{k} \neq 0$ for $k=$ $1,2, \ldots$, and $[0, \omega] \cap\left\{t_{k}\right\}=\left\{t_{1}, t_{2}, \ldots, t_{m}\right\}$; hence $q=m$. The main purpose of this paper is to derive easily verifiable sufficient conditions for the existence of multiple positive periodic solutions of (2).

\section{Preliminaries}

In this section, we cite some definitions and lemmas.

Let $P C_{\omega}$ denote the space of $\omega$-periodic functions $\psi$ : $\mathbb{R} \rightarrow \mathbb{R}$ which are continuous for $t \neq t_{k}$, are continuous from the left for $t \in \mathbb{R}$, and have possible discontinuities of the first kind at points $t=t_{k}$; that is, the limit from the right of $t_{k}$ exists but may be different from the value at $t_{k}$. We also denote $P C_{\omega}^{1}=\left\{\psi \in P C_{\omega}: \dot{\psi} \in P C_{\omega}\right\}$. lemmas.

For the convenience, we list the following definitions and

Definition 1 (see [17]). The set $\mathbb{F} \subset P C_{\omega}$ is said to be quasiequicontinuous in $[0, \omega]$ if for any $\epsilon>0$ there exists a $\delta>0$ such that if $x \in \mathbb{F}, k \in \mathbb{Z}, \tau_{1}, \tau_{2} \in\left(t_{k-1}, t_{k}\right) \cap[0, \omega]$, and $\left|\tau_{1}-\tau_{2}\right|<\delta$, then

$$
\left|x\left(\tau_{1}\right)-x\left(\tau_{2}\right)\right|<\epsilon .
$$

Lemma 2 (see [17]). The set $\mathbb{F} \subset P C_{\omega}$ is relatively compact if and only if

(1) $\mathbb{F}$ is bounded, that is, $\|x\| \leq M$, for each $x \in \mathbb{F}$, and some $M>0$;

(2) $\mathbb{F}$ is quasiequicontinuous in $[0, \omega]$.

Lemma 3 (see [17]). Assume that $\psi \in P C_{\omega}^{1}$; then the following inequality holds:

$$
\begin{aligned}
& \sup _{s \in[0, \omega]} \psi(s)-\inf _{s \in[0, \omega]} \psi(s) \\
& \quad \leq \frac{1}{2}\left[\int_{0}^{\omega}|\dot{\psi}(s)| d s+\sum_{k=1}^{m}\left|\Delta \psi\left(t_{k}\right)\right|\right] .
\end{aligned}
$$

\section{Existence of Multiple Positive Periodic Solutions}

In this section, sufficient conditions are obtained for the existence of periodic solutions of (2).

In order to obtain the existence of positive periodic solutions of (2), for convenience, we will summarize in the following a few concepts and results from [8] that will be basic for this section.
Let $X, Y$ be normed vector spaces, let $L: \operatorname{Dom} L \subset X \rightarrow$ $Y$ be a linear mapping, and let $N: X \rightarrow Y$ be a continuous mapping. The mapping $L$ is called a Fredholm mapping of index zero if $\operatorname{dimKer} L=\operatorname{codim} \operatorname{Im} L<+\infty$ and $\operatorname{Im} L$ is closed in $Y$. If $L$ is a Fredholm mapping of index zero and there exist continuous projectors $P: X \rightarrow X$ and $Q: Y \rightarrow$ $Y$ such that $\operatorname{Im} P=\operatorname{Ker} L$ and $\operatorname{Ker} Q=\operatorname{Im} L=\operatorname{Im}(I-Q)$, it follows that $L \mid \operatorname{dom} L \cap \operatorname{Ker} P:(I-P) X \rightarrow \operatorname{Im} L$ is invertible. We denote the inverse of that map by $K_{p}$. If $\Omega$ is an open bounded subset of $X$, the mapping $N$ will be called $L$ compact on $\bar{\Omega}$ if $Q N(\bar{\Omega})$ is bounded and $K_{p}(I-Q) N: \bar{\Omega} \rightarrow$ $X$ is compact. Since $\operatorname{Im} Q$ is isomorphic to $\operatorname{Ker} L$, there exists an isomorphism $J: \operatorname{Im} Q \rightarrow \operatorname{Ker} L$.

Lemma 4 (see [8]). Let $\Omega \subset X$ be an open and bounded set. Let $L$ be a Fredholm mapping of index zero and let $N$ be $L$ compact on $\bar{\Omega}$. Assume,
(a) for each $\lambda \in(0,1)$ and $x \in \partial \Omega \cap \operatorname{Dom} L, L x \neq \lambda N x$;
(b) for each $x \in \partial \Omega \cap \operatorname{Ker} L, Q N x \neq 0$;
(c) $\operatorname{deg}\{J Q N, \Omega \cap \operatorname{Ker} L, 0\} \neq 0$.

Then $L x=N x$ has at least one solution in $\bar{\Omega} \cap \operatorname{Dom} L$.

Before starting our main result, for the lack of convenience, we denote

$$
\begin{aligned}
& \bar{f}=\frac{1}{\omega} \int_{0}^{\omega} f(t) \mathrm{d} t \quad f \in P C_{\omega}, \\
& c_{j}=\sum_{k=1}^{m} \ln \left(1+c_{j k}\right), \quad j=1,2, \\
& C_{1}=\sum_{k=1}^{m}\left|\ln \left(1+c_{1 k}\right)\right|+c_{1}, \\
& C_{2}=\sum_{k=1}^{m}\left|\ln \left(1+c_{2 k}\right)\right|-c_{2} .
\end{aligned}
$$

The following assumptions are valid throughout this paper:

$$
\begin{aligned}
& \left(A_{1}\right) \bar{d}-\left(c_{2} / \omega\right)>0, \\
& \left(A_{2}\right) 1 / 4 h<G<1 / 3 h, \\
& \left(A_{3}\right) 4 h\left(\bar{d}-\left(c_{2} / \omega\right)\right) \exp \left[(1 / 2)\left(2 \bar{r} \omega+C_{1}\right)\right]<\bar{B}< \\
& 4 G h^{2}\left(\bar{d}-\left(c_{2} / \omega\right)\right) /(4 G h-1) .
\end{aligned}
$$


For further convenience, we introduce six positive numbers as follows:

$$
\begin{gathered}
h_{ \pm}=\left(\left[e \delta \bar{B} \exp \left(-\frac{1}{2}\left(2 \bar{r} \omega+C_{1}\right)\right)\right.\right. \\
\left.\left.-2 h e \delta\left(\bar{d}-\frac{c_{2}}{\omega}\right)\right] \pm \sqrt{\Delta_{1}}\right) \\
\times\left(2\left(\bar{d}-\frac{c_{2}}{\omega}\right) h^{2} e^{2} \delta^{2}\right)^{-1}, \\
l_{ \pm}=\left(\left[4 G h^{2} e \delta \bar{B} \exp \left(\frac{1}{2}\left(2 \bar{r} \omega+C_{1}\right)\right)\right.\right. \\
\times\left(2 h^{2} e^{2} \delta^{2}\left[4 G h^{2}\left(\bar{d}-\frac{c_{2}}{\omega}\right)-(4 G h-1) \bar{B}\right]\right)^{-1} \\
u_{ \pm}=\frac{\left[4 G e \delta \bar{B}-8 G h e \delta\left(\bar{d}-\left(c_{2} / \omega\right)\right)\right] \pm \sqrt{\Delta_{3}}}{2\left[4 G h^{2} e^{2} \delta^{2}\left(\bar{d}-\left(c_{2} / \omega\right)\right)-(4 G h-1) e^{2} \delta^{2} \bar{B}\right]},
\end{gathered}
$$

where

$$
\begin{aligned}
\Delta_{1}= & {\left[e \delta \bar{B} \exp \left(-\frac{1}{2}\left(2 \bar{r} \omega+C_{1}\right)\right)-2 h e \delta\left(\bar{d}-\frac{c_{2}}{\omega}\right)\right]^{2} } \\
& -4 h^{2} e^{2} \delta^{2}\left(\bar{d}-\frac{c_{2}}{\omega}\right)^{2}, \\
\Delta_{2}= & {\left[4 G h^{2} e \delta \bar{B} \exp \left(\frac{1}{2}\left(2 \bar{r} \omega+C_{1}\right)\right)\right.} \\
& \left.-2 h e \delta\left[4 G h^{2}\left(\bar{d}-\frac{c_{2}}{\omega}\right)-(4 G h-1) \bar{B}\right]\right]^{2} \\
& -4 h^{2} e^{2} \delta^{2}\left[4 G h^{2}\left(\bar{d}-\frac{c_{2}}{\omega}\right)-(4 G h-1) \bar{B}\right]^{2} \\
\Delta_{3}= & {\left[4 G e \delta \bar{B}-8 G h e \delta\left(\bar{d}-\frac{c_{2}}{\omega}\right)\right]^{2} } \\
& -16 G\left(\bar{d}-\frac{c_{2}}{\omega}\right)\left[4 G h^{2} e^{2} \delta^{2}\left(\bar{d}-\frac{c_{2}}{\omega}\right)\right. \\
& \left.\quad-(4 G h-1) e^{2} \delta^{2} \bar{B}\right] .
\end{aligned}
$$

Under assumptions $\left(A_{1}\right),\left(A_{2}\right)$, and $\left(A_{3}\right)$, it is not difficult to show that

$$
l_{-}<u_{-}<h_{-}<h_{+}<u_{+}<l_{+} \text {. }
$$

Theorem 5. In addition to $\left(A_{1}\right),\left(A_{2}\right)$, and $\left(A_{3}\right)$, suppose that

$$
\left(A_{4}\right) \bar{r}+\left(c_{1} / \omega\right)-(\bar{r} / K) \exp \left(\ln l_{+}+(1 / 2)\left(2 \bar{r} \omega+C_{1}\right)\right)>0 .
$$

Then system (2) has at least two positive $\omega$-periodic solutions.

Proof. Making the change of variables,

$$
N(t)=\exp \left(u_{1}(t)\right), \quad P(t)=\exp \left(u_{2}(t)\right) .
$$

Then, system (2) can be rewritten as

$$
\begin{gathered}
\dot{u}_{1}(t)=r(t)-\frac{r(t)}{K} \exp \left(u_{1}\left(t-\tau_{1}\right)\right) \\
-\frac{4 G e \delta \exp \left(u_{2}(t)\right)+(4 G h-1) e^{2} \delta^{2} \exp \left(u_{1}(t)+u_{2}(t)\right)}{4 G\left(1+h e \delta \exp \left(u_{1}(t)\right)\right)^{2}} \\
\equiv f_{1}(t), \\
\begin{aligned}
\dot{u}_{2}(t)=-d(t) \\
+\left(\left(4 G e \delta B(t) \exp \left(u_{1}\left(t-\tau_{2}\right)\right)\right.\right. \\
\left.+(4 G h-1) e^{2} \delta^{2} B(t) \exp \left(2 u_{1}(t)\right)\right) \\
\left.\times\left(4 G\left(1+h e \delta \exp \left(u_{1}(t)\right)\right)^{2}\right)^{-1}\right) \\
\equiv f_{2}(t), \quad t \neq t_{k}, \\
\Delta u_{1}\left(t_{k}\right)=\ln \left(1+c_{1 k}\right), \\
\Delta u_{2}\left(t_{k}\right)=\ln \left(1+c_{2 k}\right), \quad t=t_{k} .
\end{aligned}
\end{gathered}
$$

Take

$$
\begin{aligned}
& X=\left\{x=\left(u_{1}, u_{2}\right)^{T}: u_{j} \in P C_{\omega}, j=1,2,\right. \\
&x(t+\omega)=x(t)\}, \quad Y=X \times \mathbb{R}^{2 q}
\end{aligned}
$$

and define

$$
\begin{gathered}
\|x\|_{0}=\sum_{j=1}^{2} \sup _{t \in[0, \omega]}\left|u_{j}(t)\right|, \quad x=\left(u_{1}, u_{2}\right) \in X, \\
\|y\|_{1}=\|x\|_{0}+\sum_{j=1}^{q}\left\|\xi_{j}\right\|, \quad y=\left[x, \xi_{1}, \ldots, \xi_{q}\right] \in Y .
\end{gathered}
$$

$\operatorname{Both}(X,\|\cdot\|)$ and $\left(Y,\|\cdot\|_{1}\right)$ are Banach spaces.

Define

$\operatorname{Dom} L=\{x \in X: \dot{x} \in X\}, \quad L: \operatorname{Dom} L \longrightarrow Y$,

$$
L\left(\left(u_{1}, u_{2}\right)^{T}\right)=\left(\left(\begin{array}{l}
\dot{u}_{1} \\
\dot{u}_{2}
\end{array}\right),\left(\begin{array}{c}
\Delta u_{1}\left(t_{k}\right) \\
\Delta u_{2}\left(t_{k}\right)
\end{array}\right)_{k=1}^{q}\right) ;
$$

$N: X \rightarrow Y$,

$$
N\left(\begin{array}{l}
u_{1} \\
u_{2}
\end{array}\right)=\left(\left(\begin{array}{l}
f_{1}(t) \\
f_{2}(t)
\end{array}\right),\left(\begin{array}{l}
\ln \left(1+c_{1 k}\right) \\
\ln \left(1+c_{2 k}\right)
\end{array}\right)_{k=1}^{q}\right) ;
$$

$P: X \rightarrow X, P\left(\left(u_{1}, u_{2}\right)^{T}\right)=\left(\overline{u_{1}}, \overline{u_{2}}\right)^{T} ; Q: Y \rightarrow Y$,

$$
\begin{aligned}
& Q\left(\left(\begin{array}{l}
u_{1} \\
u_{2}
\end{array}\right),\left\{\left(\begin{array}{l}
m_{k} \\
n_{k}
\end{array}\right)\right\}_{k=1}^{q}\right)
\end{aligned}
$$

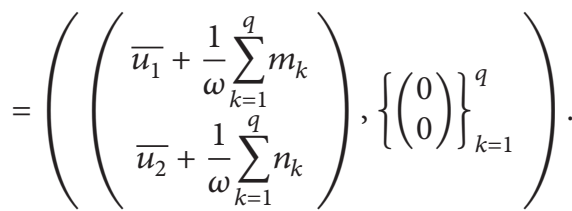


It is not difficult to show that

$\operatorname{Ker} L=\left\{x \mid x \in X,\left(u_{1}, u_{2}\right)^{T} \in X:\left(u_{1}(t), u_{2}(t)\right)^{T}=\right.$ $\left.\left(c_{1}, c_{2}\right)^{T} \in \mathbb{R}^{2}, t \in \mathbb{R}\right\}$;

$$
\operatorname{Im} L=\left\{\left[\left(\begin{array}{l}
u_{1} \\
u_{2}
\end{array}\right),\left\{\left(\begin{array}{c}
m_{k} \\
n_{k}
\end{array}\right)\right\}_{k=1}^{q}\right] \in Y: \quad \begin{array}{c}
\omega \overline{u_{1}}+\sum_{k=1}^{q} m_{k}=0 \\
\omega \overline{u_{2}}+\sum_{k=1}^{q} n_{k}=0
\end{array}\right\} .
$$

Since $\operatorname{Im} L$ is closed in $Y$ and $P$ and $Q$ are continuous projectors such that

$$
\begin{gathered}
\operatorname{Im} P=\operatorname{Ker} L, \quad \operatorname{Ker} Q=\operatorname{Im} L=\operatorname{Im}(I-Q), \\
\operatorname{dim} \operatorname{Ker} L=\operatorname{codim} \operatorname{Im} L=2,
\end{gathered}
$$

it follows that $L$ is a Fredholm mapping of index zero. Furthermore, the generalized inverse (to $L$ ) $K_{p}: \operatorname{Im} L \rightarrow$ Dom $L \cap \operatorname{Ker} P$ exists, which is given by

$$
\begin{aligned}
K_{p}\left[\left(\begin{array}{l}
u_{1} \\
u_{2}
\end{array}\right),\left\{\left(\begin{array}{l}
m_{k} \\
n_{k}
\end{array}\right)\right\}_{k=1}^{q}\right] \\
=\left(\begin{array}{c}
\int_{0}^{t} u_{1}(s) \mathrm{d} s+\sum_{0<t_{k}<t} m_{k}-\frac{1}{\omega} \sum_{k=1}^{q} m_{k}-\overline{\int_{0}^{t} u_{1}(s) \mathrm{d} s} \\
\int_{0}^{t} u_{2}(s) \mathrm{d} s+\sum_{0<t_{k}<t} n_{k}-\frac{1}{\omega} \sum_{k=1}^{q} n_{k}-\overline{\int_{0}^{t} u_{2}(s) \mathrm{d} s}
\end{array}\right) .
\end{aligned}
$$

Then $Q N: X \rightarrow Y$ and $K_{p}(I-Q) N: X \rightarrow X$ are defined by

$$
\begin{aligned}
Q N\left(\begin{array}{l}
u_{1} \\
u_{2}
\end{array}\right)= & \left.\left(\begin{array}{l}
\overline{f_{1}}+\frac{c_{1}}{\omega} \\
\overline{f_{2}}+\frac{c_{2}}{\omega}
\end{array}\right),\left\{\left(\begin{array}{l}
0 \\
0
\end{array}\right)\right\}_{k=1}^{q}\right) . \\
K_{p}(I-Q) N\left(\begin{array}{l}
u_{1} \\
u_{2}
\end{array}\right)= & \left(\begin{array}{l}
\int_{0}^{t} f_{1}(s) \mathrm{d} s+\sum_{0<t_{k}<t} \ln \left(1+c_{1 k}\right) \\
\int_{0}^{t} f_{2}(s) \mathrm{d} s+\sum_{0<t_{k}<t} \ln \left(1+c_{2 k}\right)
\end{array}\right) \\
& +\left(\frac{1}{2}-\frac{t}{\omega}\right)\left(\begin{array}{l}
\int_{0}^{\omega} f_{1}(t) \mathrm{d} t+c_{1} \\
\int_{0}^{\omega} f_{2}(t) \mathrm{d} t+c_{2}
\end{array}\right) \\
& -\frac{1}{\omega}\left(\begin{array}{c}
\int_{0}^{\omega} \int_{0}^{t} f_{1}(s) \mathrm{d} s \mathrm{~d} t+c_{1} \\
\int_{0}^{t} f_{0}(s) \mathrm{d} s \mathrm{~d} t+c_{2}
\end{array}\right) .
\end{aligned}
$$

Clearly, QN and $K_{p}(I-Q) N$ are continuous. By using the Arzela-Ascoli theorem, it is not difficult to prove that $\overline{K_{p}(I-Q) N(\bar{\Omega})}$ is compact for any open bounded set $\Omega \subset X$.
Moreover, $\mathrm{QN}(\bar{\Omega})$ is bounded. Therefore, $N$ is $L$-compact on $\bar{\Omega}$ with any open bounded set $\Omega \subset X$.

Now, we reach the position to search for an appropriate open, bounded subset $\Omega$ for the application of the continuation theorem.

Corresponding to the operator equation, $L x=\lambda N x, \lambda \epsilon$ $(0,1)$, we have

$$
\dot{u}_{1}(t)=\lambda\left[r(t)-\frac{r(t)}{K} \exp \left(u_{1}\left(t-\tau_{1}\right)\right)\right.
$$

$$
\begin{gathered}
-\left(\left(4 G e \delta \exp \left(u_{2}(t)\right)+(4 G h-1) e^{2} \delta^{2}\right.\right. \\
\left.\times \exp \left(u_{1}(t)+u_{2}(t)\right)\right) \\
\left.\left.\times\left(4 G\left(1+h e \delta \exp \left(u_{1}(t)\right)\right)^{2}\right)^{-1}\right)\right], \\
\dot{u}_{2}(t)=\lambda\left[-d(t)+\left(\left(4 G e \delta B(t) \exp \left(u_{1}\left(t-\tau_{2}\right)\right)\right.\right.\right. \\
\left.+(4 G h-1) e^{2} \delta^{2} B(t) \exp \left(2 u_{1}(t)\right)\right) \\
\left.\left.\times\left(4 G\left(1+h e \delta \exp \left(u_{1}(t)\right)\right)^{2}\right)^{-1}\right)\right], \\
\Delta \neq t_{k} \\
\Delta u_{1}\left(t_{k}\right)=\lambda \ln \left(1+c_{1 k}\right), \\
\Delta u_{2}\left(t_{k}\right)=\lambda \ln \left(1+c_{2 k}\right), \quad t=t_{k} .
\end{gathered}
$$

Suppose that $x=\left(u_{1}(t), u_{2}(t)\right)^{T} \in X$ is a solution of (21) for a certain $\lambda \in(0,1)$. Integrating the first equation of (21) over the interval $[0, \omega]$, we obtain

$$
\begin{aligned}
& \int_{0}^{\omega} \frac{r(t)}{K} \exp \left(u_{1}\left(t-\tau_{1}\right)\right) \mathrm{d} t \\
& \quad+\int_{0}^{\omega} \frac{4 G e \delta \exp \left(u_{2}(t)\right)+(4 G h-1) e^{2} \delta^{2} \exp \left(u_{1}(t)+u_{2}(t)\right)}{4 G\left(1+h e \delta \exp \left(u_{1}(t)\right)\right)^{2}} \mathrm{~d} t \\
& =\bar{r} \omega+c_{1} .
\end{aligned}
$$

Similarly, integrating the second equation of (21) over the interval $[0, \omega]$, we obtain

$$
\begin{aligned}
& \int_{0}^{\omega}\left(4 G e \delta B(t) \exp \left(u_{1}\left(t-\tau_{2}\right)\right)\right. \\
& \left.\quad+(4 G h-1) e^{2} \delta^{2} B(t) \exp \left(2 u_{1}(t)\right)\right) \\
& \quad \times\left(4 G\left(1+h e \delta \exp \left(u_{1}(t)\right)\right)^{2}\right)^{-1} \mathrm{~d} t=\bar{d} \omega-c_{2} .
\end{aligned}
$$

It follows from the first equation of (21) and (22) and $\left(A_{2}\right)$ that

$$
\begin{aligned}
& \int_{0}^{\omega}\left|\dot{u}_{1}(t)\right| \mathrm{d} t \\
& \quad=\lambda \int_{0}^{\omega} \mid\left[r(t)-\frac{r(t)}{K} \exp \left(u_{1}\left(t-\tau_{1}\right)\right)\right.
\end{aligned}
$$




$$
\begin{aligned}
&-(\left(4 G e \delta \exp \left(u_{2}(t)\right)+(4 G h-1) e^{2} \delta^{2}\right. \\
&\left.\times \exp \left(u_{1}(t)+u_{2}(t)\right)\right) \\
&\left.\left.\times\left(4 G\left(1+h e \delta \exp \left(u_{1}(t)\right)\right)^{2}\right)^{-1}\right)\right] \mid \mathrm{d} t \\
&<\int_{0}^{\omega} r(t) \mathrm{d} t+\int_{0}^{\omega} \frac{r(t)}{K} \exp \left(u_{1}\left(t-\tau_{1}\right)\right) \mathrm{d} t \\
&+\int_{0}^{\omega}\left(4 G e \delta \exp \left(u_{2}(t)\right)+(4 G h-1) e^{2} \delta^{2}\right. \\
&\left.\times \exp \left(u_{1}(t)+u_{2}(t)\right)\right) \\
& \times\left(4 G\left(1+h e \delta \exp \left(u_{1}(t)\right)\right)^{2}\right)^{-1} \mathrm{~d} t \\
&=\int_{0}^{\omega} r(t) \mathrm{d} t+\bar{r} \omega+c_{1}=2 \bar{r} \omega+c_{1} ;
\end{aligned}
$$

that is,

$$
\int_{0}^{\omega}\left|\dot{u}_{1}(t)\right| \mathrm{d} t<2 \bar{r} \omega+c_{1} .
$$

Similarly, it follows from the second equation of (21) and (23) and $\left(A_{2}\right)$ that

$$
\int_{0}^{\omega}\left|\dot{u}_{2}(t)\right| \mathrm{d} t<2 \bar{d} \omega-c_{2}
$$

Since $\left(u_{1}(t), u_{2}(t)\right)^{T} \in X$, there exists $\xi_{i}, \eta_{i} \in[0, \omega]$, such that

$$
u_{i}\left(\xi_{i}\right)=\inf _{t \in[0, \omega]} u_{i}(t), \quad u_{i}\left(\eta_{i}\right)=\sup _{t \in[0, \omega]} u_{i}(t), \quad i=1,2 .
$$

From $\left(A_{2}\right)$ and (23), we see that

$$
\begin{aligned}
\bar{d} \omega-c_{2} \leq & \int_{0}^{\omega} \frac{4 G e \delta B(t) \exp \left(u_{1}\left(t-\tau_{2}\right)\right)}{4 G\left(1+h e \delta \exp \left(u_{1}(t)\right)\right)^{2}} \mathrm{~d} t \\
& +\int_{0}^{\omega} \frac{(4 G h-1) e^{2} \delta^{2} B(t) \exp \left(2 u_{1}(t)\right)}{4 G h^{2} e^{2} \delta^{2} \exp \left(2 u_{1}(t)\right)} \mathrm{d} t
\end{aligned}
$$

which implies

$$
\bar{d}-c_{2} \leq \frac{e \delta \bar{B} \exp \left(u_{1}\left(\eta_{1}\right)\right)}{\left(1+h e \delta \exp \left(u_{1}\left(\xi_{1}\right)\right)\right)^{2}}+\frac{(4 G h-1) \bar{B}}{4 G h^{2}}
$$

So

$$
\begin{aligned}
& u_{1}\left(\eta_{1}\right) \\
& \geq \ln \frac{\left[4 G h^{2}\left(\bar{d}-\frac{c_{2}}{\omega}\right)-(4 G h-1) \bar{B}\right]\left(1+h e \delta \exp \left(u_{1}\left(\xi_{1}\right)\right)\right)^{2}}{4 G h^{2} e \delta \bar{B}} .
\end{aligned}
$$

This, combined with Lemma 3, gives

$$
\begin{aligned}
u_{1}\left(\xi_{1}\right)+\frac{1}{2}(2 \bar{r} \omega & \left.+C_{1}\right) \\
>\ln ( & \left(\left[4 G h^{2}\left(\bar{d}-\frac{c_{2}}{\omega}\right)-(4 G h-1) \bar{B}\right]\right. \\
& \left.\times\left(1+h e \delta \exp \left(u_{1}\left(\xi_{1}\right)\right)\right)^{2}\right) \\
& \left.\times\left(4 G h^{2} e \delta \bar{B}\right)^{-1}\right)
\end{aligned}
$$

or equivalently

$$
\begin{gathered}
{\left[4 G h^{2}\left(\bar{d}-\frac{c_{2}}{\omega}\right)-(4 G h-1) \bar{B}\right] h^{2} e^{2} \delta^{2} \exp \left(2 u_{1}\left(\xi_{1}\right)\right)} \\
-\left[4 G h^{2} e \delta \bar{B} \exp \left(\frac{1}{2}\left(2 \bar{r} \omega+C_{1}\right)\right)\right. \\
-2 h e \delta\left(4 G h^{2}\left(\bar{d}-\frac{c_{2}}{\omega}\right)\right. \\
-(4 G h-1) \bar{B})] \exp \left(u_{1}\left(\xi_{1}\right)\right) \\
+\left[4 G h^{2}\left(\bar{d}-\frac{c_{2}}{\omega}\right)-(4 G h-1) \bar{B}\right]<0 .
\end{gathered}
$$

In view of $\left(A_{1}\right)$ and $\left(A_{3}\right)$ we have

$$
\ln l_{-}<u_{1}\left(\xi_{1}\right)<\ln l_{+} .
$$

Similarly, it follows from $\left(A_{2}\right)$ and (23) that

$$
\bar{d} \omega-c_{2} \geq \int_{0}^{\omega} \frac{4 G e \delta B(t) \exp \left(u_{1}\left(t-\tau_{2}\right)\right)}{4 G\left(1+h e \delta \exp \left(u_{1}(t)\right)\right)^{2}} \mathrm{~d} t,
$$

which implies

$$
\bar{d}-\frac{c_{2}}{\omega} \geq \frac{e \delta \bar{B} \exp \left(u_{1}\left(\xi_{1}\right)\right)}{\left(1+h e \delta \exp \left(u_{1}\left(\eta_{1}\right)\right)\right)^{2}} .
$$

So

$$
u_{1}\left(\xi_{1}\right) \leq \ln \frac{\left(\bar{d}-\left(c_{2} / \omega\right)\right)\left(1+h e \delta \exp \left(u_{1}\left(\eta_{1}\right)\right)\right)^{2}}{e \delta \bar{B}} .
$$

This, combined with Lemma 3, gives

$$
\begin{aligned}
& u_{1}\left(\eta_{1}\right)-\frac{1}{2}\left(2 \bar{r} \omega+C_{1}\right) \\
&<\ln \frac{\left(\bar{d}-\left(c_{2} / \omega\right)\right)\left(1+h e \delta \exp \left(u_{1}\left(\eta_{1}\right)\right)\right)^{2}}{e \delta \bar{B}}
\end{aligned}
$$

or equivalently

$$
\begin{aligned}
& \left(\bar{d}-\frac{c_{2}}{\omega}\right) h^{2} e^{2} \delta^{2} \exp \left(2 u_{1}\left(\eta_{1}\right)\right) \\
& \quad-\left[e \delta \bar{B} \exp \left(-\frac{1}{2}\left(2 \bar{r} \omega+C_{1}\right)\right)-2 h e \delta\left(\bar{d}-\frac{c_{2}}{\omega}\right)\right] \\
& \quad \times \exp \left(u_{1}\left(\eta_{1}\right)\right)+\left(\bar{d}-\frac{c_{2}}{\omega}\right)>0 .
\end{aligned}
$$


It follows from $\left(A_{1}\right)$ and $\left(A_{3}\right)$ that

$$
u_{1}\left(\eta_{1}\right)<\ln h_{-} \text {or } u_{1}\left(\eta_{1}\right)>\ln h_{+} .
$$

It follows from (25), (33), and Lemma 3 that

$$
u_{1}\left(\eta_{1}\right) \leq \ln l_{+}+\frac{1}{2}\left(2 \bar{r} \omega+C_{1}\right) \triangleq H_{11}
$$

On the other hand, it follows from $\left(A_{2}\right)$ and (22) and (40) that

$$
\begin{aligned}
\bar{r} \omega+c_{1} \geq \int_{0}^{\omega} & \frac{4 G e \delta \exp \left(u_{2}\left(\xi_{2}\right)\right)}{4 G\left(1+h e \delta \exp \left(H_{11}\right)\right)^{2}} \mathrm{~d} t \\
\bar{r} \omega+c_{1} \leq & \int_{0}^{\omega} \frac{r(t)}{K} \exp \left(H_{11}\right) \mathrm{d} t \\
& +\int_{0}^{\omega} e \delta \exp \left(u_{2}\left(\eta_{2}\right)\right) \mathrm{d} t \\
& +\int_{0}^{\omega} \frac{e \delta \exp \left(u_{2}\left(\eta_{2}\right)\right)}{2} \mathrm{~d} t .
\end{aligned}
$$

It follows from (41) that

$$
u_{2}\left(\xi_{2}\right) \leq \ln \frac{\left(\bar{r}+\left(c_{1} / \omega\right)\right)\left(1+h e \delta \exp \left(H_{11}\right)\right)^{2}}{e \delta}
$$

This, combined with Lemma 3, gives

$$
\begin{aligned}
u_{2}\left(\eta_{2}\right) \leq & \ln \frac{\left(\bar{r}+\left(c_{1} / \omega\right)\right)\left(1+h e \delta \exp \left(H_{11}\right)\right)^{2}}{e \delta} \\
& +\frac{1}{2}\left(2 \bar{d} \omega+C_{2}\right) \triangleq H_{21} .
\end{aligned}
$$

Moreover, because of $\left(A_{4}\right)$, it follows from (42) that

$$
u_{2}\left(\eta_{2}\right) \geq \ln \frac{2\left[\bar{r}+\left(c_{1} / \omega\right)-(\bar{r} / K) \exp \left(H_{11}\right)\right]}{3 e \delta}
$$

This, combined with Lemma 3 again, gives

$$
\begin{aligned}
u_{2}\left(\xi_{2}\right) \geq & \ln \frac{2\left[\bar{r}+\left(c_{1} / \omega\right)-(\bar{r} / K) \exp \left(H_{11}\right)\right]}{3 e \delta} \\
& -\frac{1}{2}\left(2 \bar{d} \omega+C_{2}\right) \triangleq H_{22} .
\end{aligned}
$$

It follows from (44) and (46) that

$$
\sup _{t \in[0, \omega]}\left|u_{2}(t)\right|<\max \left\{\left|H_{21}\right|,\left|H_{22}\right|\right\} \triangleq H_{2} .
$$

Obviously, $\ln l_{ \pm}, \ln h_{ \pm}, H_{11}$, and $H_{2}$ are independent of $\lambda$.
Now, let us consider $Q N x$ with $X=\left(u_{1}, u_{2}\right)^{T} \in \mathbb{R}^{2}$. Note that

$$
\begin{aligned}
& \text { QN }\left(u_{1}, u_{2}\right) \\
& =\left[\bar{r}-\frac{\bar{r}}{K} \exp \left(u_{1}\right)\right. \\
& \quad-\frac{4 G e \delta \exp \left(u_{2}\right)+(4 G h-1) e^{2} \delta^{2} \exp \left(u_{1}+u_{2}\right)}{4 G\left(1+h e \delta \exp \left(u_{1}\right)\right)^{2}}+\frac{c_{1}}{\omega}, \\
& -\bar{d}+\frac{4 G e \delta \bar{B} \exp \left(u_{1}\right)+(4 G h-1) e^{2} \delta^{2} \bar{B} \exp \left(2 u_{1}\right)}{4 G\left(1+h e \delta \exp \left(u_{1}\right)\right)^{2}} \\
& \left.+\frac{c_{2}}{\omega}\right] .
\end{aligned}
$$

Because of $\left(A_{1}\right),\left(A_{2}\right),\left(A_{3}\right)$, and $\left(A_{4}\right)$, we can show that the equation $Q N\left(u_{1}, u_{2}\right)=0$ has two distinct solutions:

$$
\begin{aligned}
& \tilde{u}=\left(\ln u_{-}, \ln \frac{4 G\left(\bar{r}-(\bar{r} / K) u_{-}\right)\left(1+h e \delta u_{-}\right)^{2}}{4 G e \delta+(4 G h-1) e^{2} \delta^{2} u_{-}}\right), \\
& \widehat{u}=\left(\ln u_{+}, \ln \frac{4 G\left(\bar{r}-(\bar{r} / K) u_{+}\right)\left(1+h e \delta u_{+}\right)^{2}}{4 G e \delta+(4 G h-1) e^{2} \delta^{2} u_{+}}\right) .
\end{aligned}
$$

Choose $C>0$ such that

$$
\begin{aligned}
C>\max & \left\{\left|\ln \frac{4 G\left(\bar{r}-(\bar{r} / K) u_{-}\right)\left(1+h e \delta u_{-}\right)^{2}}{4 G e \delta+(4 G h-1) e^{2} \delta^{2} u_{-}}\right|,\right. \\
& \left.\left|\ln \frac{4 G\left(\bar{r}-(\bar{r} / K) u_{+}\right)\left(1+h e \delta u_{+}\right)^{2}}{4 G e \delta+(4 G h-1) e^{2} \delta^{2} u_{+}}\right|\right\} .
\end{aligned}
$$

Let

$$
\begin{aligned}
& \Omega_{1}=\left\{x=\left(u_{1}, u_{2}\right)^{T} \in X \mid \begin{array}{l}
u_{1}(t) \in\left(\ln l_{-}, \operatorname{In} h_{-}\right) \\
\sup _{t \in[0, \omega]}\left|u_{2}(t)\right|<H_{2}+C
\end{array}\right\}, \\
& \Omega_{2}=\left\{x=\left(u_{1}, u_{2}\right)^{T} \in X \mid \begin{array}{r}
\inf _{t \in[0, \omega]} u_{1}(t) \in\left(\ln l_{-}, \operatorname{In} l_{+}\right) \\
\sup _{t \in[0, \omega]} u_{1}(t) \in\left(\ln h_{+}, H_{11}\right) \\
\sup _{t \in[0, \omega]}\left|u_{2}(t)\right|<H_{2}+C
\end{array}\right\} .
\end{aligned}
$$

Then both $\Omega_{1}$ and $\Omega_{2}$ are bounded open subsets of $X$. It follows from (2) and (50) that $\tilde{u} \in \Omega_{1}$ and $\widehat{u} \in \Omega_{2}$. With the help of (2), (33), (39), (40), and (47)-(50), it is easy to see that $\Omega_{1} \cap \Omega_{2}=\phi$ and $\Omega_{i}$ satisfies the requirement (a) in Lemma 4 for $i=1,2$. Moreover, $Q N x \neq 0$ for $x \in \partial \Omega \cap \operatorname{Ker} L=\partial \Omega \cap$ $\mathbb{R}^{2}$. A direct computation gives $\operatorname{deg}\left\{J Q N, \Omega_{i} \cap \operatorname{Ker} L, 0\right\} \neq 0$. Here, $J$ is taken as the identity mapping since $\operatorname{Im} Q=\operatorname{Ker} L$. So far we have proved that $\Omega_{i}$ satisfies all the assumptions in Lemma 4. Hence, Equation (2) has at least two $\omega$-periodic solutions. This completes the proof of Theorem 5 . 


\section{Conflict of Interests}

The authors declare that there is no conflict of interests regarding the publication of this paper.

\section{References}

[1] Y. Kuang, Delay Differential Equations with Application in Population Dynamics, vol. 191, Academic Press, New York, NY, USA, 1993.

[2] H. I. Freedman, Deterministic Mathematical Models in Population Ecology, vol. 57, Marcel Dekker, New York, NY, USA, 1980.

[3] C. S. Holling, "The functional response of predator to prey density and its role in mimicry and population regulation," Memoirs of the Entomological Society of Canada, vol. 97, supplement 45, pp. 5-60, 1965.

[4] R. S. Liu, Z. L. Feng, H. Zhu, and D. L. DeAngelis, "Bifurcation analysis of a plant-herbivore model with toxin-determined functional response," Journal of Differential Equations, vol. 245, no. 2, pp. 442-467, 2008.

[5] Y. F. Gao, P. Wong, Y. Xia, and X. Yuan, "Multiple periodic solutions of a nonautonomous Plant-Hare model," Abstract and Applied Analysis, vol. 2014, Article ID 130856, 7 pages, 2014.

[6] Y.-H. Xia, X. Chen, and V. G. Romanovski, "On the linearization theorem of Fenner and Pinto," Journal of Mathematical Analysis and Applications, vol. 400, no. 2, pp. 439-451, 2013.

[7] Y.-H. Xia, J. Li, and P. J. Y. Wong, "On the topological classification of dynamic equations on time scales," Nonlinear Analysis. Real World Applications. An International Multidisciplinary Journal, vol. 14, no. 6, pp. 2231-2248, 2013.

[8] R. E. Gaines and J. L. Mawhin, Coincidence Degree and Nonlinear Differential Equations, Springer, Berlin, Germany, 1977.

[9] Y. H. Xia, J. Cao, and S. S. Cheng, "Multiple periodic solutions of a delayed stage-structured predator-prey model with nonmonotone functional responses," Applied Mathematical Modelling, vol. 31, no. 9, pp. 1947-1959, 2007.

[10] F. Y. Wei, "Existence of multiple positive periodic solutions to a periodic predator-prey system with harvesting terms and Holling III type functional response," Communications in Nonlinear Science and Numerical Simulation, vol. 16, no. 4, pp. 2130-2138, 2011.

[11] Y.-H. Xia, "Global asymptotic stability of an almost periodic nonlinear ecological model," Communications in Nonlinear Science and Numerical Simulation, vol. 16, no. 11, pp. 4451-4478, 2011.

[12] Y. H. Xia, "Global analysis of an impulsive delayed LotkaVolterra competition system," Communications in Nonlinear Science and Numerical Simulation, vol. 16, no. 3, pp. 1597-1616, 2011.

[13] Y.-H. Xia, "Periodic solution of certain nonlinear differential equations: via topological degree theory and matrix spectral theory," International Journal of Bifurcation and Chaos in Applied Sciences and Engineering, vol. 22, no. 8, Article ID 1250196, 17 pages, 2012.

[14] D. D. Bainov and P. S. Simeonov, Impulsive Differential Equations: Asymptotic Properties of the Solution, vol. 28, World Scientific, Singapore, 1995.

[15] D. Bainov and P. Simeonov, Impulsive Differential Equations: Periodic Solutions and Applications, vol. 66, Longman Scientific \& Technical, New York, NY, USA, 1993.
[16] V. Lakshmikantham, D. D. Baĭnov, and P. S. Simeonov, Theory of Impulsive Differential Equations, vol. 6, World Scientific, Singapore, 1989.

[17] Q. Wang, B. X. Dai, and Y. M. Chen, "Multiple periodic solutions of an impulsive predator-prey model with Holling-type IV functional response," Mathematical and Computer Modelling, vol. 49, no. 9-10, pp. 1829-1836, 2009. 


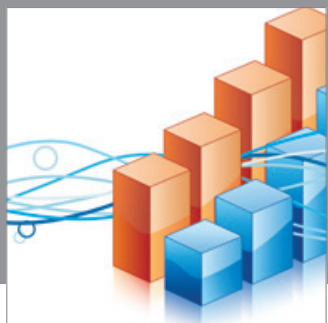

Advances in

Operations Research

mansans

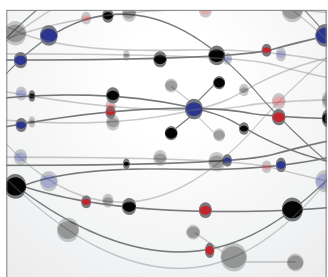

The Scientific World Journal
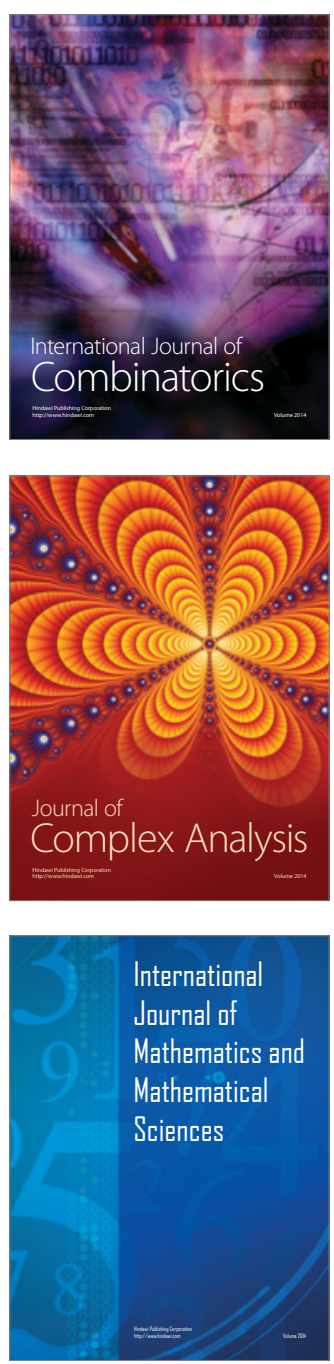
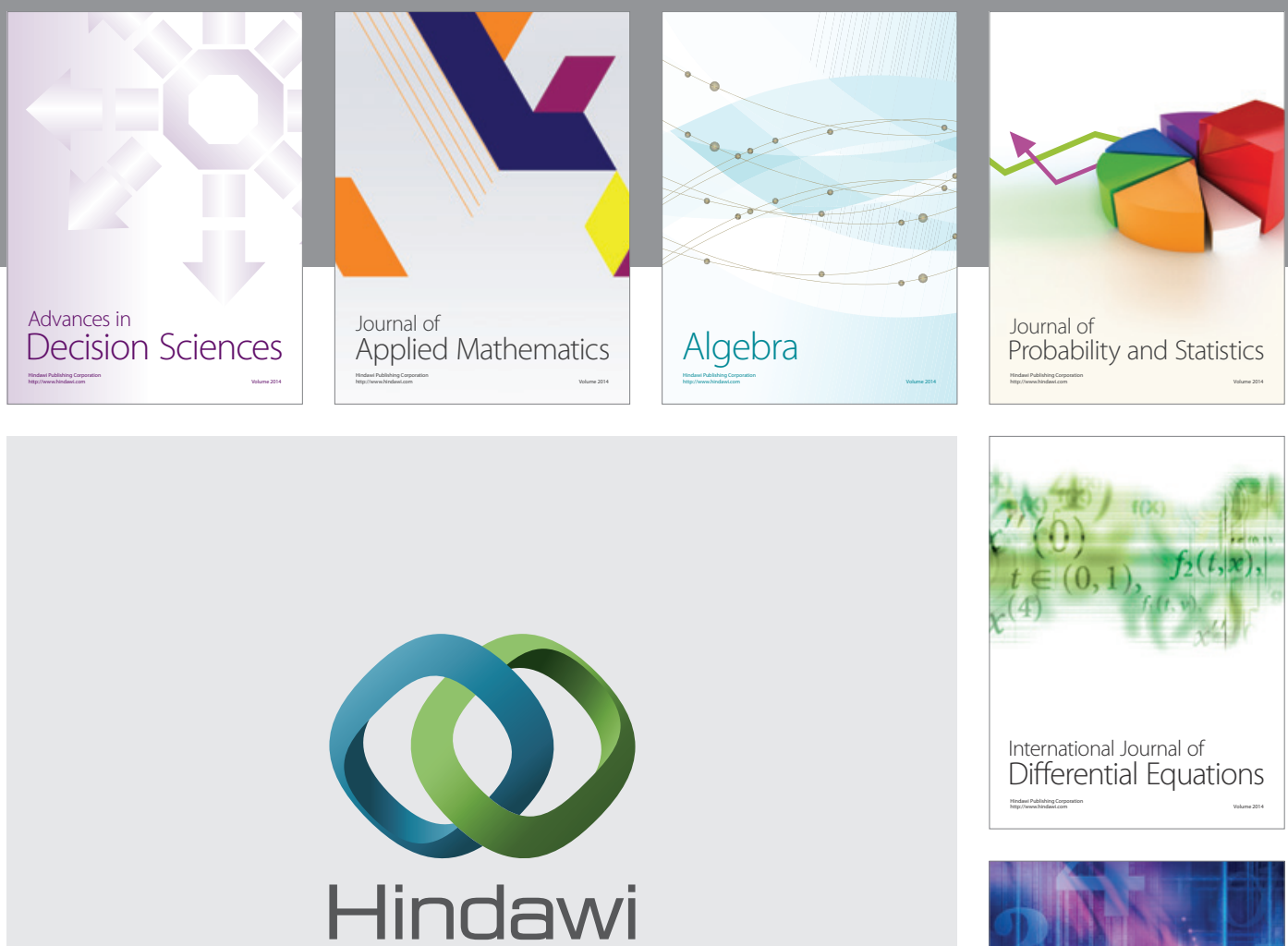

Submit your manuscripts at http://www.hindawi.com
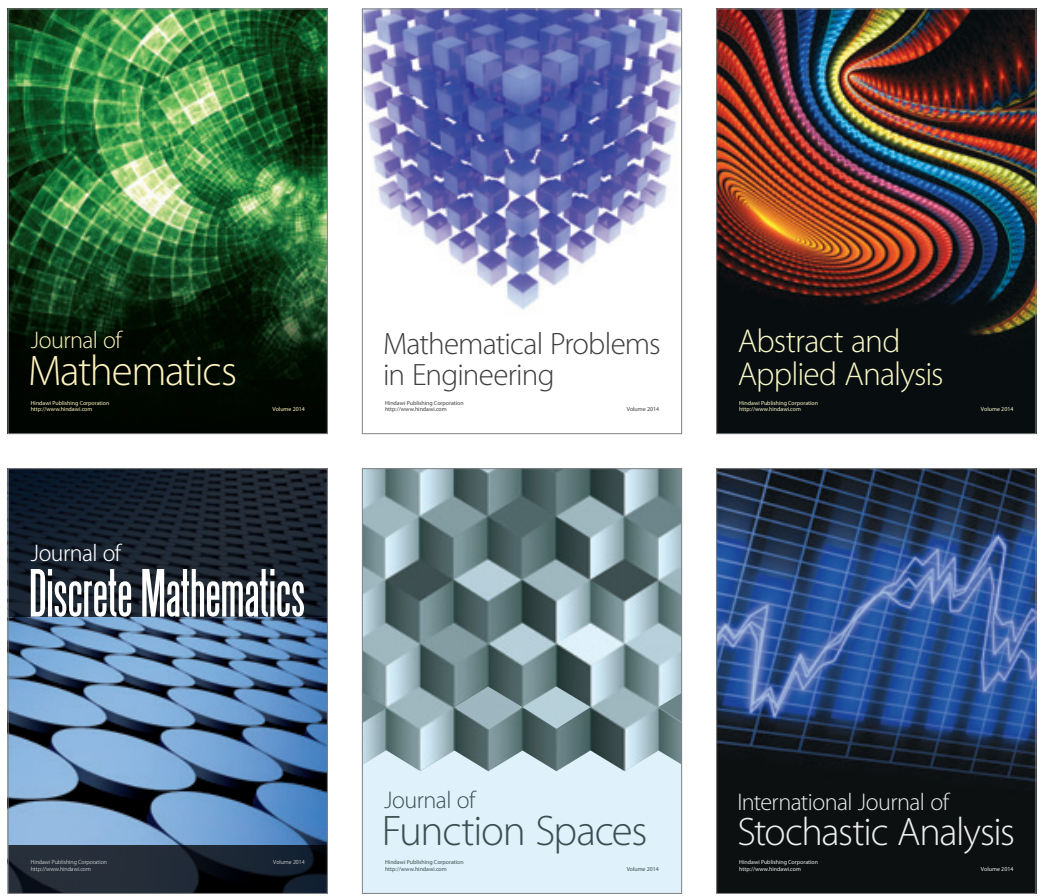

Journal of

Function Spaces

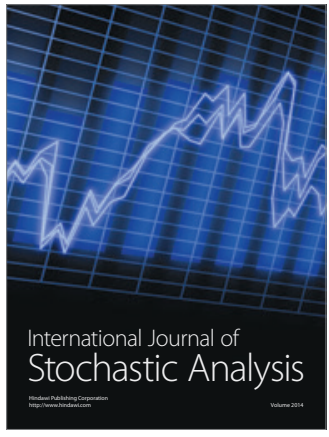

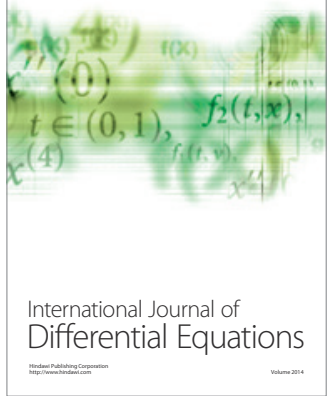
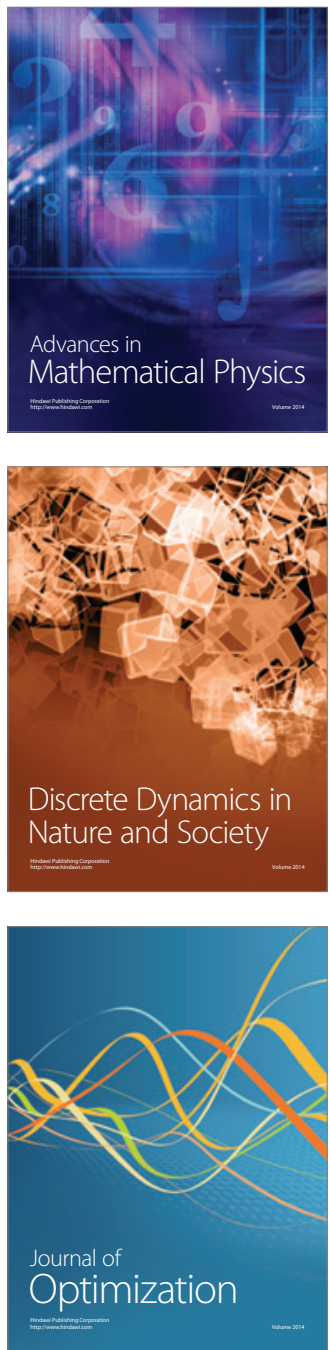